\title{
The Newly Diagnosed Myeloma Patient - Therapeutic Approaches
}

\author{
Jesús San-Miguel \\ Professor of Medicine, and Head, Haematology Department, University Hospital of Salamanca
}

DOI: 10.17925/EOH.2010.06.1.81

\begin{abstract}
The outcome for myeloma patients has significantly improved over the last decade, mainly due to the introduction of new drugs with a singular mechanism of action such as thalidomide and lenalidomide (revlimid), both immunomodulatory drugs (IMIDS), and the proteasome inhibitor bortezomib (velcade). In newly diagnosed young patients, induction regimens such as bortezomib-thalidomide or lenalidomide plus dexamethasone will replace vincristine, doxorubicin and dexamethasone (VAD), followed by high-dose melphalan. Maintenance with IMIDs may represent the new standard of care for young multiple myeloma (MM) patients. This approach is being challenged by continuous treatment with novel agents and the postponement of a transplant until relapse. Allogeneic transplant should be conducted within the context of clinical trials. In elderly patients or non-transplant candidates, new regimens based on melphalan-prednisone plus thalidomide, velcade or lenalidomide have become new standards. The reduction of side effects while maintaining efficacy of treatment is most important in the elderly population.
\end{abstract}

\section{Keywords}

Thalidomide, lenalidomide, bortezomib, autologous transplant, allogeneic transplant, side effects, thrombosis, peripheral neuropathy, complete response

Disclosure: Jesús San-Miguel has participated in advisory boards for Millennium, Celgene and Janssen-Cilag.

Received: 28 September 2009 Accepted: 16 March 2010 Citation: European Oncology, 2010;6(1):81-4

Correspondence: Jesús San-Miguel, Servicio de Hematología, Hospital Universitario de Salamanca, Paseo de San Vicente, 58, 37007 Salamanca, Spain. E: sanmigiz@usal.es

The outcome for myeloma patients has significantly improved over the last decade, mainly due to the introduction of new drugs with a singular mechanism of action, such as thalidomide and lenalidomide (revlimid), both immunomodulatory drugs (IMIDS), and the proteasome inhibitor bortezomib (velcade). ${ }^{1}$ In this article, we will briefly review the treatment of newly diagnosed patients stratified according to age (above or below 65-70 years of age) and transplant or non-transplant candidate status.

\section{Treatment of Newly Diagnosed Transplant Candidate Patients Induction}

The combination of vincristine, doxorubicin and dexamethasone (VAD) has long been the gold standard as a preparatory regimen for young newly diagnosed multiple myeloma (MM) patients who are candidates for autologous stem cell transplant (ASCT), with partial response (PR) rates ranging between 52 and $63 \%$ and complete response (CR) rates of $3-13 \%$. However, novel drug combinations appear to be superior to VAD, such as regimens to decrease tumour burden pre-transplant (TrX). Three randomised trials have compared thalidomide ( $\mathrm{T}$ )-based regimens $(\mathrm{T}+$ Dex or TAD or T + VAD) versus either high-dose DEX or VAD as an initial therapy in transplant-eligible patients. ${ }^{2-4}$ In all studies, thalidomide combinations were superior to conventional induction treatment, although the response rate (>PR) obtained with $\mathrm{T}+$ Dex (63\%) was lower than that achieved with TAD or T + VAD $(80 \%$, but CR rates are usually $<10 \%)$. The MRC group has compared cyclosphophamide + TD versus cyclophosphamide + VAD (CVAD) as an induction regimen before transplant; the thalidomide arm was significantly superior, with a response rate (RR) of 87 versus $75 \%$ including 20 versus $12 \%$ CRS, respectively. In studies evaluating bortezomib (Bz) combination therapy, data from both a French randomised trial comparing Bz + Dex versus VAD and an Italian trial comparing Bz-TD versus TD show superiority of Bz regimens both before and after transplant, with 80 versus $94 \%>P R$ including 15 versus $32 \% \mathrm{CR}$, respectively. These results are consistent with several previous pilot studies. In terms of lenalidomide (Len), two large randomised studies have shown that the majority of patients $(>85 \%)$ respond to Len + Dex induction, but probably a minimum of six cycles would be required to achieve a substantial number of CRs. ${ }^{5}$ Novel agents did not affect stem cell collection, although for lenalidomide it is recommended to collect the cells after no more than four to six cycles using cyclophosphamide with G-CSF. In summary, current results indicate that novel induction regimens are superior to VAD.

\section{Autologous Stem Cell Transplant}

High-dose therapy (HDT) (usually based on melphalan 200mg/m²) followed by ASCT prolonged overall survival (OS) compared with standard-dose therapy (SDT) in prospective randomised trials conducted by French (Francophone Myeloma Intergroup [IFM]) and English (Medica Research Council [MRC]) groups and has provided evidence for a more than 10-year survival rate in at least a subset of patients. ${ }^{6,7}$ Nevertheless, the US study (Southwest Oncology Group [SWOG] 9321), the French MAG91 study and the Spanish PETHEMA-94 trial, although confirmed the benefit of ASCT in terms of response rate and event-free survival (EFS), did not find superiority in terms of survival compared with SDT., These discrepancies can be, at least in part, explained by: differences in study design (the Spanish study randomised patients responding to initial therapy while, in the others, randomisation was performed 
up-front); differences in the conditioning regimens; and, particularly, differences in the intensity and duration of the chemotherapy arm (the dose of alkylating agents and steroids were higher in the SWOG and Spanish trials, which may explain why OS for conventionally treated patients was longer in these two studies compared with the IFM and MRC trials. Despite these discrepancies, HDT is currently considered the standard of care for younger patients with MM, mainly based on the benefit on response rate and EFS.

In the setting of novel agents, it is also important to define whether or not ASCT enhances the response rates obtained with these new induction regimens. Studies based on bortezomib combinations, including two randomised trials, have shown that the CR rate was improved following ASCT, suggesting that induction with novel agents and ASCT is a complementary rather than alternative treatment approach. Moreover, with TAD, Bz-Dex and Bz-TD, preliminary data already show a prolongation in PFS. Nevertheless, longer follow-up is needed. Some investigators argue that the 'induction with novel agents followed by $\mathrm{ASCT}^{\prime}$ approach may be challenged by the optimal results obtained with 'long-term' treatment with novel combinations (i.e. Len + Dex). Although a randomised trial comparing these two approaches would be most interesting, the transplant approach induces a very high $\mathrm{CR}$ rate (a goal in all haematological malignancies) and patients enjoy a long-term period free of treatment with an excellent quality of life.

Regarding tandem $\mathrm{ASCT}^{10-12}$ its use will decrease for two reasons: according to the IFM and Italian experiences, only patients achieving a less than 'very good' partial response with the first transplant benefit from the second; and a similar benefit is obtained upon using thalidomide as consolidation/maintenance therapy. By contrast, a second transplant at relapse may be increasingly used, provided that the duration of the response to first transplant has lasted for more than two to three years.

\section{Maintenance}

The third step in this sequence of treatment is maintenance. Interferon and/or corticosteroids have shown little benefit and have been abandoned. The availability of novel agents (particularly those in oral formulations - thalidomide and lenalidomide) has renewed the concept of maintenance in an attempt to prolong the duration of response after transplant. ${ }^{13-15}$ The IFM group has shown that thalidomide maintenance after tandem ASCT is significantly superior to no maintenance or pamidronate alone in terms of EFS (52, 36 and $37 \%$ at three years, respectively) and OS (87 versus $74 \%$ at four years). This superiority has been confirmed by an Australian study comparing thalidomide plus prednisone versus prednisone alone. Of note, the Arkansas group has also observed that the use of thalidomide as part of the induction and maintenance phase was associated with longer EFS, but only translated into an OS benefit for a subset of patients with high-risk cytogenetics. Similarly, the MMRC group has reported a short survival after relapse for patients receiving maintenance with thalidomide. This raises an important concern about whether the continuous use of novel agents may induce more resistant relapses. Moreover, the benefit of thalidomide maintenance for patients who are already in $\mathrm{CR}$, as well as for those with poor cytogenetics, is not well established: the IMF and MMRC groups showed no benefit for patients with deletion 13q and 17p (detected by fluorescent in situ hybridisation [FISH] analysis), respectively, while, as mentioned above, in the Arkansas study there was a survival advantage for patients with abnormal cytogenetics treated with thalidomide. Accordingly, although randomised trials are required to define the role of long-term maintenance (greater than one year), a short treatment with thalidomide would be justified in patients who have not achieved CR post-ASCT. Moreover, it is possible that the better tolerance of lenalidomide will facilitate its use in maintenance programmes.

\section{Allogeneic Transplant}

Allogeneic transplantation remains the only curative therapeutic approach in MM patients. However, it is associated with a high transplant-related mortality (TRM) of up to $30-50 \%$ as well as morbidity, mainly due to chronic graft-versus-host disease (GVHD). Accordingly, it should be used in carefully defined situations and, preferably, within the context of clinical trials. In order to decrease TRM, different reduced-intensity conditioning regimens (RIC) have been developed (allo-RIC), mainly based on fludarabine and melphalan or fludarabine plus radiotherapy (2Gy). ${ }^{16-18}$ TRM decreases to $15-25 \%$, but this is associated with a higher incidence of relapses. In a prospective randomised trial, the French group compared double ASCT with ASCT followed by allo-RIC among patients displaying poor prognostic features (high B2 microglobuline and monosomy of chromosome). Unfortunately, there were no event-free survivors at five years either after double ASCT or after ASCT followed by allo-RIC. Similar results have been reported by the Hovon group. By contrast, both the Italian and the EBMT groups, using a similar approach, have described an improvement in terms of OS among patients receiving ASCT followed by allo-RIC compared with double autologous transplant. The Spanish group has recently reported a trend towards a longer PFS in favour of allo-RIC, but with a similar OS. Differences in patient characteristics, GVHD prophylaxis and conditioning regimens could contribute to explaining these discrepant results. Moreover, unfortunately, a high proportion of patients develop extra-medullary relapses without bone marrow involvement, indicating that, although the disease may be under control in the bone marrow milieu, extramedullary spread may occur.

In order to use allo-Trx as rescue therapy, a prerequisite is to obtain either complete remission or a 'very good' partial response before transplant, as most patients with active disease will not benefit from this procedure. Once again, transplants should be performed by experienced groups and within clinical trials. Donor lymphocyte infusions (DLI) given for relapsed myeloma following allogeneic transplantation induce responses in $30-50 \%$ of patients, but unfortunately the long-term efficacy is limited. Interestingly, the combination of DLI with thalidomide, lenalidomide or bortezomib may improve the response rate and contribute to modulating the immune response, although further studies are required to confirm these data.

\section{Treatment Options for the Elderly Patient Not Suitable for Stem Cell Transplantation}

Melphalan + prednisone (MP) has been the gold standard for over 40 years; however, recent results based on the combination of MP with either thalidomide or bortezomib, and probably also with lenalidomide, indicate that there are now new standards of care for elderly MM patients. ${ }^{19-21}$

Five randomised trials have compared T+MP (MPT) versus MP. In all these studies both the RR and CRs were significantly higher in the MPT arm (RR $57-76 \%$ for MPT versus $31-48 \%$ for MP, CR $7-16 \%$ for 
MPT versus $1-4 \%$ for MP). Moreover, the five studies showed a significant advantage of MPT treatment in terms of PFS/TTP, (prolongation in PFS ranging from two to nine months). However, only in the two French studies was MPT treatment associated with a significant prolongation in OS. The toxicity associated with the high dose of thalidomide used in some of these trials may contribute to explaining the survival discrepancies. Preliminary data from the MRC myeloma IX trial shows that the combination of cyclophosphamide + thalidomide + dexamethasone (CTD) is superior to MP in terms of RR (82 versus 49\%) and CRs (22 versus 6\%), respectively. MP has also been compared with thalidomide + dexamethasone (TD) and, although the RR was higher in the experimental arm, the OS was shorter. It should be noted that in this last trial TD treatment was associated with a higher rate of early discontinuations due to toxicity, and higher mortality, particularly during the first year.

Lenalidomide has also been combined with MP. A pilot study showed an $81 \%$ RR, with $17 \%$ CR and an EFS of $87 \%$ at 16 months. A large randomised trial comparing Len + MP versus MP has recently been completed. Nevertheless, MP combinations are being challenged by the recent results reported with Len + Dex, particularly using low-dose Dex, with a two-year survival probability of $82 \%$ in patients $>65$ years of age and very good tolerability.

The proteasome inhibitor bortezomib has been tested in combination with MP in a pilot study conducted by the Spanish group; the positive results have recently been confirmed in a large (682-patient) randomised study (VISTA), which compared BzMP versus MP. The RR for BzMP versus MP were 71 versus $35 \%$ with 30 versus $4 \% C R$, respectively. BzMP treatment was associated with a $52 \%$ reduction in the risk of progression, with a median TTP of 24 versus 16.6 months $(p<0.0001)$ and a $36 \%$ reduction in risk of death, which translates into an OS at three years of 72 versus 59\% for BzMP versus MP. This benefit was present in spite of $45 \%$ of the MP patients having received bortezomib upon progression. In addition, time to next therapy and treatment-free interval were significantly prolonged in patients treated with BzMP (28.1 and 16.1 months versus 20.8 and 9.8 months, respectively), and the benefit of this combination was observed across all patient subgroups. An updated analysis shows that survival after relapsing from VMP and MP continues to show a significant benefit in favour of VMP patients, which indicates that frontline bortezomib use does not induce more resistant relapses. Preliminary data from ongoing trials with modified VMP schemes (using weekly doses of Btz) suggest that the tolerability can be significantly improved while maintaining efficacy.

A final controversial matter is whether there is any preference for one of the three novel MP combinations. An individualised treatment approach would probably be valuable because:

- the most mature data are with MPT and BzMP;

- for patients with antecedent or risk of deep venous thrombosis (DVT), BzMP could be the preferable option;

- in patients with antecedent peripheral neuropathy, Len-MP should be the choice;

- in patients with renal insufficiency, BzMP is safe;

- in patients living long distances from hospital, oral treatment (MPT or Len-MP) would be preferable; and

- in patients with poor compliance with treatment, BzMP could be better.
In patients $>75$ years of age or with a fragile condition, it would be recommended to use modified regimens, with a lower dose of thalidomide (100mg), lenalidomide (15-20mg) or bortezomib (1 $\mathrm{mg} / \mathrm{m}^{2}$ or a weekly schedule). One additional possibility in these patients is to substitute melphalan with cyclophosphamide (50mg/day or $1 \mathrm{~g} / 21$ days), as this latter agent is less myelotoxic. In very elderly patients, special attention must be paid to infectious episodes (requiring active treatment) and renal function (needing appropriate hydration), particularly during the first three months of treatment when they are responsible for the high incidence of early deaths.

A range of novel agent combinations are now available, which have demonstrated superior efficacy over the traditional MP regimen, indicating that MP should no longer be considered the standard treatment in this population. Ongoing studies will establish optimal dosing and treatment schedules for different populations with the aim of maximising efficacy and improving tolerability.

\section{Side Effects Associated with Novel Agents}

Due to the previous history of thalidomide, a major concern was the toxicity profile. The side effects are dose-related, and the most common are constipation, weakness, somnolence and neuropathy. Peripheral neuropathy is a common adverse event with thalidomide therapy, and often limits both the dose and duration of treatment. The use of combination therapy has raised concerns about an increased risk of DVT. Apparently, the major risk of DVT occurs when tumour load is high and thalidomide is combined with chemotherapy, especially adriamycin (20\% incidence compared with $4 \%$ when thalidomide is used alone). Accordingly, in this setting, anticoagulant prophylaxis with lowmolecular-weight heparin (LMWH) or aspirin is mandatory. Current data suggest that lenalidomide is better tolerated than thalidomide in several aspects - it does not usually produce clinically significant somnolence, constipation or neuropathy, although the incidence of myelosuppresion is higher, mainly neutropenia (G3 in 17-30\%) and thrombocytopenia, which are manageable with dose reduction and growth factor support. Similar to thalidomide, lenalidomide is associated with a higher risk of DVT (5-25\%) and the risk increases in patients with co-morbidities, previous history of DVT, concomitant use of erythropoietin, high-dose Dex, anthracyclines or high tumour mass. For these reasons, anticoagulant prophylaxis with LMWH or aspirin is mandatory. The most frequent G3 toxicities of bortezomib included fatigue, gastrointestinal symptoms (G3 in 15-25\%), cyclical thrombocytopenia and, particularly, peripheral neuropathy. This last side effect, emerging as G3 in 6-15\% of the patients, is the main reason for treatment discontinuation, and the early detection of PN is most important in order to reduce the dose or frequency of injections. Nevertheless, it resolved or improved in twothirds of patients after completion or discontinuation of therapy.

Jesús San-Miguel is a Professor of Medicine (Haematology) and Head of the Haematology Department at the University Hospital of Salamanca in Spain. He is a member of the advisory board of the International Myeloma Foundation, the Multiple Myeloma Research Foundation and the Carreras Foundation and a board member of the Spanish Haematology and Genome Foundations. He is member of the Editorial Board of several scientific journals and Associate Editor of Haematologica. He was Vice Director of the Cancer Research Centre in Salamanca, and as Chairman of the Spanish Myeloma Group (GEM) he organised the IXth International Myeloma Workshop held in Salamanca in 2003. Professor San-Miguel served the European Haematology Association (EHA) as a board councillor and Chairman of the scientific committee of the ninth congress (2004) and President of the XI EHA Congress. He has received numerous prizes, including the Waldenström Award in 2007 and Spanish prizes for both oncology and translational research. He has published over 450 original papers in international journals. 
1. San-Miguel J, Harousseau JL, Joshua D, Anderson KC, Individualizing treatment of patients with myeloma in the era of novel agents, J Clin Oncol, 2008;26:2761-2766.

2. Rajkumar SV, Rosiñol L, Hussein M, et al., Multicenter, randomized, double-blind, placebo-controlled study of thalidomide plus dexamethasone compared with dexamethasone as initial therapy for newly diagnosed multiple myeloma, J Clin Oncol, 2008;26:2171-2177.

3. Cavo M, Zamagni E, Tosi P, et al., Superiority of thalidomide and dexamethasone over vincristinedoxorubicin-dexamethasone (VAD) as primary therapy in preparation for autologous transplantation for multiple myeloma, Blood, 2005:106:35-39.

4. Lokhorst HM, Schmidt-Wolf I, Sonneveld P, et al., Thalidomide in induction treatment increases the very good partial response rate before and after high-dose therapy in previously untreated multiple myeloma, Haematologica, 2008:93:124-127.

5. Niesvizky R, Jayabalan DS, Christos PJ, et al., BiRD (Biaxin [clarithromycin]/Revlimid [lenalidomide]/dexamethasone) combination therapy results in high complete- and overall-response rates in treatment-naive symptomatic multiple myeloma, Blood, 2008:111:1101-1109.

6. Attal M, Harousseau JL, Stoppa AM, et al., A prospective randomized trial of autologous bone marrow transplantation and chemotherapy in multiple myeloma. Intergroupe Francais du Myelome, N Engl J Med 1996;335:91-97.

7. Child JA, Morgan GJ, Davies FE, et al., High-dose chemotherapy with hematopoietic stem-cell rescue for multiple myeloma, N Engl J Med, 2003:348:1875-1883.

8. Barlogie B, Kyle RA, Anderson KC, et al., Standard chemotherapy compared with high-dose chemoradiotherapy for multiple myeloma: final results of phase III US Intergroup Trial S9321, I Clin Oncol 2006;24:929-936.

9. Blade J, Rosinol L, Sureda A, et al., High-dose therapy intensification compared with continued standard chemotherapy in multiple myeloma patients responding to the initial chemotherapy: long-term results from a prospective randomized trial from the Spanish cooperative group PETHEMA, Blood, 2005;106:3755-3759.

10. Attal M, Harousseau JL, Facon T, et al., Single versus double autologous stem-cell transplantation for multiple myeloma, N Eng/ J Med, 2003;349:2495-2502.

11. Cavo M, Tosi P, Zamagni E, et al., Prospective, randomized study of single compared with double autologous stem-cell transplantation for multiple myeloma: Bologna 96 clinical study, I clin Oncol, 2007:25:2434-2441.

12. Attal M, Harousseau JL, Leyvraz S, et al., Maintenance therapy with thalidomide improves survival in patients with multiple myeloma, Blood, 2006;108:3289-3294.

13. Spencer A, Prince HM, Roberts AW, et al., Consolidation therapy with low-dose thalidomide and prednisolone prolongs the survival of multiple myeloma patients undergoing a single autologous stem-cell transplantation procedure, I Clin Oncol, 2009:27:1788-1793.

14. Barlogie B, Tricot G, Anaissie E, et al., Thalidomide and hematopoietic-cell transplantation for multiple myeloma N Eng/ J Med, 2006:354:1021-1030.

15. Barlogie B, Pineda-Roman $\mathrm{M}$, Van RF, et al., Thalidomid arm of Total Therapy 2 improves complete remission duration and survival in myeloma patients with metaphase cytogenetic abnormalities, Blood, 2008:112:3115-3121.

16. Garban $\mathrm{F}$, Attal $\mathrm{M}$, Michallet $\mathrm{M}$, et al., Prospective comparison of autologous stem cell transplantation followed by dose-reduced allograft (IFM99-03 trial) with tandem autologous stem cell transplantation (IFM99-04 trial) in high-risk de novo multiple myeloma, Blood, 2006;107:3474-3480

17. Bruno B, Rotta M, Patriarca F, et al., A comparison of allografting with autografting for newly diagnosed myeloma, N Engl J Med, 2007;356:1110-1120.

18. Rosinol L, Perez-Simon JA, Sureda A, et al., A prospective PETHEMA study of tandem autologous transplantation versus autograft followed by reduced-intensity conditioning allogeneic transplantation in newly diagnosed multiple myeloma, Blood, 2008;112:3591-3593.

19. Facon T, Mary JY, Hulin C, et al., Melphalan and prednisone plus thalidomide versus melphalan and prednisone alone or reduced-intensity autologous stem cell transplantation in elderly patients with multiple myeloma (IFM 99-06): a randomised trial, Lancet, 2007;370:1209-1218.

20. Palumbo A, Bringen S, Caravita T, et al., Oral melphalan and prednisone chemotherapy plus thalidomide compared with melphalan and prednisone alone in elderly patients with multiple myeloma: randomized controlled trial, Lancet, 2006;367:825-31

21. San Miguel J, Schlag R, Khuageva N, et al., A Phase 3 Study Comparing Bortezomib+Melphalan+Prednison (VMP) with Melphalan+Prednisone (MP) in Newly Diagnosed Multiple Myeloma, N Engl I Med, (2008)359(9):906-17. 